\title{
Applications of Genetic Algorithms in Realistic Wind Field Simulations
}

\author{
R. Montenegro, G. Montero, E. Rodríguez, J.M. Escobar and J.M. \\ González-Yuste \\ Institute for Intelligent Systems and Numerical Applications in Engineering, \\ University of Las Palmas de Gran Canaria, Campus Universitario de Tafira, \\ 35017 Las Palmas de Gran Canaria, Spain, rafa@dma.ulpgc.es
}

Mass consistent models have been widely use in 3-D wind modelling by finite element method. We have used a method for constructing tetrahedral meshes which are simultaneously adapted to the terrain orography and the roughness length by using a refinement/derefinement process in a 2-D mesh corresponding to the terrain surface, following the technique proposed in $[14,15,18]$. In this 2-D mesh we include a local refinement around several points which are previously defined by the user. Besides, we develop a technique for adapting the mesh to any contour that has an important role in the simulation, like shorelines or roughness length contours [3, 4], and we refine the mesh locally for improving the numerical solution with the procedure proposed in [6].

This wind model introduces new aspects on that proposed in [16, 20, 19]. The characterization of the atmospheric stability is carried out by means of the experimental measures of the intensities of turbulence. On the other hand, since several measures are often available at a same vertical line, we have constructed a least square optimization of such measures for developing a vertical profile of wind velocities from an optimum friction velocity. Besides, the main parameters governing the model are estimated using genetic algorithms with a parallel implementation $[20,12,26]$. In order to test the model, some numerical experiments are presented, comparing the results with realistic measures.

\section{Introduction}

The society has been becoming aware of environmental problems and nowadays it appreciates the use of renewable energies. Along last years the use of wind power for producing electric energy has augmented considerably. So companies of this sector are requesting more and more sophisticated tools which allow them to face the competitive and demanding market. Wind models are tools that allow the study of several problems related to the atmosphere, such 
as, the effect of wind on structures, pollutant transport, fire spreading, wind farm location, etc.

Diagnostic models are not used to make forecasts through integrating conservative relations [23]. Therefore they are also called kinetic models [9]. This models generate wind fields that satisfy some physical conditions. If mass conservation law is the only imposed equation, we are defining a mass consistent model. The relative simplicity of diagnostic models makes them attractive from the practical point of view, since they do not require many input data and may be easily used. Pennel [22] checked that, in some cases, improved mass consistent models such as NOABL and COMPLEX obtained better results than other dynamic models which are more complex and expensive. However, we have to take into account that diagnostic models neither consider thermal effects nor those due to pressure gradients. As a consequence, problems like sea breezes can not be simulated with these models unless such effects are incorporated into the initial wind data using observations made in selected locations $[8,21]$. So diagnostic models have been designed for predicting the effects of the orography on the steady average wind, i.e., average wind in intervals from 10 minutes to 1 hour. There exists a wide range of diagnostic models which have been used by scientists in problems of meteorology and air pollution.

The primitive 2-D diagnostic models did not consider the terrain orography and the vertical profile of the wind. They built an interpolated wind field taking into account only the distance from the nodes to the measurement stations and then they solved the two-dimensional elliptic problem arising from the discretization in a plane; see [33] for a 2-D adaptive finite element model with mixed formulation. Nowadays, in problems defined over complex terrain, to have high quality meshes is essential for the discretization of the studied domains. Most of the existing models uses to work with regular meshes. This strategy is impracticable for problems with complex terrain since the size of the elements must be very small in order to capture the digital information of the map. In this case, there would be regions with no fine details where such small element size would not be necessary. This would finally lead us to larger linear systems of equations and higher computational cost for solving them. Our first 3-D models (see [16, 17]) had some of these limitations since the meshes used for defining the terrain surface were uniform. In [20], we presented a new finite element model that uses adaptive nonstructured meshes of tetrahedra with elements of small size where it is necessary but maintaining greater elements where such level of discretization is not required. The resulting 3-D mesh also contains more nodes near the terrain surface, where we need more precision. In a postprocess, the mesh is smoothed and, if necessary, untangled by using the Escobar et al algorithm [3] in order to improve its quality. In addition, a local refinement procedure was proposed for improving the numerical solution [6]. Finally, although mass consistent models are widely used, they are often criticized because their results strongly depend on some governing parameters. This parameters are generally approximated us- 
ing empirical criteria. Our model includes a tool for the parameter estimation based on genetic algorithms [20].

In order to check the model with realistic data, the company Desarrollos Eólicos S.A. (DESA) has provided us with technical support about digital terrain maps related to orography and roughness length, as well as measurements of wind and turbulence intensity in some anemometers located in Lugo (Spain). This work deals with the procedures required for inserting all of this information in the wind modelling. In section 2 , we present the improvements to the adaptive discretization of the 3-D domain, including an adaptive procedure to capture the orography and roughness information simultaneously and additional local refinements in different regions of the terrain. The developed technique for inserting new information about wind measures at different heights and turbulence intensities is described in section 3. Several ideas about the parameter estimation in the wind field model is summarized in section 4 . Numerical experiments with examples that illustrate all the new possibilities of our wind model are presented in section 5 . Besides, a parameter estimation realistic problem is solved by using genetic algorithms for an episode along a day. Finally, we summarize the conclusions of this work and the topics that need further research.

\section{Adaptive Discretization of the Domain}

In this section we introduce several improvements which have been implemented in our adaptive mesh generation code: mesh adaption to terrain orography and roughness length and local refinement in the surrounding of the measurement stations or any other control point.

\subsection{Mesh Adaption to Terrain Orography and Roughness Length}

The mesh generation process starts with the determination of nodes allocated on the terrain surface. Their distribution must be adapted to the orographic and roughness characteristics in order to minimize the number of required nodes. The procedure first builds a sequence of nested meshes $T=\left\{\tau_{1}<\tau_{2}<\right.$ $\left.\ldots<\tau_{m}\right\}$ from a regular triangulation $\tau_{1}$ of the rectangular region which is studied, such that the level $\tau_{j}$ is obtained by a global refinement of the previous level $\tau_{j-1}$ with the 4-T Rivara's algorithm [25]. Every triangle of level $\tau_{j-1}$ is divided into four subtriangles inserting a new node in the middle point of the edges and connecting the node inserted in the longer edge with the opposite vertex and with the other two new nodes. Thus, in the mesh level $\tau_{j}$ there appear new nodes, edges and triangles that are defined as corresponding to level $j$. The number of levels $m$ of the sequence is determined by the degree of discretization of the terrain, i.e., the diameter of the triangulation $\tau_{m}$ must be of the order of the spacial step of the digital map that we are using (the spacial step of the roughness length map is often greater or equal to that of 
the orographic map). In this way, we ensure that this regular mesh is able to capture all the orographic and roughness information by an interpolation of the heights and roughness length in the nodes of the mesh. Finally, we define a new sequence $T^{\prime}=\left\{\tau_{1}<\tau_{2}^{\prime}<\ldots<\tau_{m^{\prime}}^{\prime}\right\}, m^{\prime} \leq m$, applying the derefinement algorithm [5, 24]. In this step, two derefinement parameters $\varepsilon_{h}$ and $\varepsilon_{r}$ are introduced and they determine the accuracy of the approximation to the terrain surface and to its roughness length, respectively. The absolute difference between the height obtained in any point of the mesh $\tau_{m^{\prime}}^{\prime}$ and the corresponding exact height will be lower than $\varepsilon_{h}$. A similar condition is established for the roughness and $\varepsilon_{r}$. A node could be eliminated only if it verifies the two derefinement conditions simultaneously.

\subsection{Local Refinement Around Control Points}

The resulting mesh adapted to orography and roughness is not always enough to ensure a prescribed accuracy of the numerical model in some regions of the domain and they may require a finner discretization. We have solve this problem by refining the terrain surface mesh in those regions such that the nodes inserted inside them are not eliminated after the derefinement procedure. The vertical spacing function and the 3-D Delaunay triangulation algorithm, that complete our 3-D mesh generator (see [14, 15, 18]), will produce a tetrahedral mesh refined around the selected regions. So, the user can define the form and location of these regions and the number of additional triangles subdivisions to be carried out inside them in order to obtain the required element size.

\section{Improvements to the Wind Model}

Our wind model has been improved in order to consider the additional information that currently may be available at measurement stations. On the one hand, we have usually different stations located in the same tower for minimizing costs and fixing the wind profiles. Thus, the computation of the friction velocity, which was directly computed from a single wind velocity measured at a station, must be obtained from several measures. For this purpose, a least square approximation is carried out. On the other hand, these stations usually provides measures of the turbulence intensity which is related to the atmospheric stability of the region. So, the knowledge of the range of turbulence intensity will allow us to select the stability class. Following the Pasquill model for the atmospheric stability [27] and defining new ranges of turbulence intensity, a new table for Pasquill stability classification is built.

\subsection{New Computation of the Friction Velocity}

We consider a log-linear profile [10] in the planetary boundary layer, which takes into account the horizontal interpolation [16], the effect of roughness 
length on the wind speed and direction, and the atmospheric stability (neutral, stable or unstable) following the Pasquill classification. In the surface layer a logarithmic wind profile is constructed,

$$
\mathbf{v}_{0}(z)=\frac{\mathbf{v}^{*}}{k}\left(\ln \frac{z}{z_{0}}-\Phi_{m}\right) \quad z_{0}<z \leq z_{s l}
$$

where $\mathbf{v}_{0}$ is the wind velocity, $k \simeq 0.4$ is the von Karman constant, $z$ is the height of the considered point over the terrain level, $z_{0}$ is the roughness length, $\Phi_{m}$ is a function depending on the atmospheric stability and $z_{s l}$ is the height of the surface layer. The friction velocity $\mathbf{v}^{*}$ is obtained at each point from the interpolation of measures at the height of the stations $z_{e}$ (horizontal interpolation),

$$
\mathbf{v}^{*}=\frac{k \mathbf{v}_{0}\left(z_{e}\right)}{\ln \frac{z_{e}}{z_{0}}-\Phi_{m}\left(z_{e}\right)}
$$

Evidently, if $n$ measures were available in a vertical line, the above equation would yield $n$ different friction velocities,

$$
\mathbf{v}_{i}^{*}=\frac{k \mathbf{v}_{0_{i}}\left(z_{e_{i}}\right)}{\ln \frac{z_{e_{i}}}{z_{0}}-\Phi_{m}\left(z_{e_{i}}\right)} \quad i=1, \ldots, n
$$

In order to obtain the optimum value of $\mathbf{v}^{*}$, we solve a least square problem involving the wind velocities measured at different height and considering that the friction velocity is not a function of the height. Consider

$$
A_{i}=\frac{1}{k}\left(\ln \frac{z_{e_{i}}}{z_{0}}-\Phi_{m}\left(z_{e_{i}}\right)\right) \quad i=1, \ldots, n
$$

such that

$$
\mathbf{v}_{0_{i}}\left(z_{e_{i}}\right)=\mathbf{v}^{*} A_{i} \quad i=1, \ldots, n
$$

If $\mathbf{v}_{s_{i}}$ is the measured velocity at the i-th station, then the function to be minimized is,

$$
F_{o b j}=\sum_{i=1}^{n}\left(\mathbf{v}_{0_{i}}\left(z_{e_{i}}\right)-\mathbf{v}_{s_{i}}\left(z_{e_{i}}\right)\right)^{2}=\sum_{i=1}^{n}\left(\mathbf{v}^{*} A_{i}-\mathbf{v}_{s_{i}}\left(z_{e_{i}}\right)\right)^{2}
$$

whose minimum is obtained for the following friction velocity,

$$
\mathbf{v}^{*}=\frac{\sum_{i=1}^{n} A_{i} \mathbf{v}_{s_{i}}\left(z_{e_{i}}\right)}{\sum_{i=1}^{n} A_{i}^{2}}
$$




\begin{tabular}{cccccc}
\multicolumn{4}{c}{ Pasquill stability class } \\
\hline \hline \multicolumn{3}{c}{ Isolation } & \multicolumn{2}{c}{ Nighttime } \\
\hline $\begin{array}{c}\text { Surface wind } \\
\text { speed (m/s) }\end{array}$ & Strong & Moderate & Slight & $\geq 4 / 8$ & $\leq 3 / 8$ \\
\hline$<2$ & Clouds & Clouds \\
\hline $2-3$ & A-B & A-B & B & - & - \\
$3-5$ & B & B-C & C & E & D \\
$5-6$ & C & C-D & D & D & D \\
$>6$ & C & D & D & D & D
\end{tabular}

For A-B, take the average of the values of $\mathrm{A}$ and $\mathrm{B}$, etc.

Table 1. Pasquill Stability Classification depending on the surface wind speed and the isolation. Strong isolation corresponds to a sunny afternoon of the middlesummer in England; slight isolation is related to same conditions in middle-winter. Nighttime means the time from one hour before the sunset to one hour after the sun rises. Neutral class D should be used also, independently of the wind speed, for clouded sky along the day or the night, and for any condition of the sky during the hour before and after the nighttime.

\subsection{Atmospheric Stability Versus Turbulence Intensity}

The atmospheric stability may be characterized by using the Pasquill stability classification of table 1 . It considers the following classes for stability: A (extremely unstable), B (moderately unstable), C (slightly unstable), D (neutral), E (slightly stable) and F (moderately stable) [27].

The anemometers generally provides measures of the intensity of turbulence that may help to complete the information about the class of atmospheric stability in the studied region. The intensity of turbulence $i$ is defined as the square root of the sum of variances $\sigma_{u}^{2}, \sigma_{v}^{2}, \sigma_{w}^{2}$, of the three components of the velocity $u_{0}, v_{0}, w_{0}$, respectively, divided by the average wind velocity that has been measured,

$$
i=\frac{\sqrt{\sigma_{u}^{2}+\sigma_{v}^{2}+\sigma_{w}^{2}}}{\left|\mathbf{v}_{0}\right|}
$$

However, only measures of speed variations are often available but not of the wind direction. In such cases, equation (8) is reduced to,

$$
i=\frac{\sigma_{v_{0}}}{\left|\mathbf{v}_{0}\right|}
$$

where $\sigma_{v_{0}}$ represents the standard deviation of the measured wind speeds.

While an unstable atmosphere implies a high level of turbulence, with a range of turbulence intensities between 0.2 and 0.4 approximately, a stable atmosphere, with a small or almost null turbulence, is characterized by intensities from 0.05 to 0.1 [13]. In table 2 , the above relations of the turbulence 
Pasquill stability class

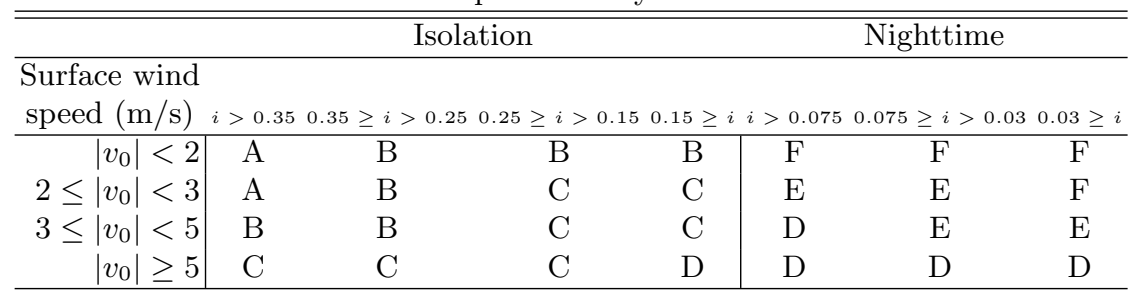

Table 2. Pasquill stability classification taking into account the surface wind speed and the turbulence stability.

intensity and the atmospheric stability have been considered in order to define the Pasquill stability class.

\section{Parameters Estimation with Genetic Algorithms}

Genetic algorithms (GAs) are optimisation tools based on the natural evolution mechanism $[12,1,30]$. They produce successive trials that have an increasing probability to obtain a global optimum. This work is based on the model developed by Levine [11]. It is a standard genetic algorithm code (pgapack library), with string real coding. The most important aspects of GAs are the construction of an initial population, the evaluation of each individual in the fitness function, the selection of the parents of the next generation, the crossover of those parents to create the children, and the mutation to increase diversity.

Two population replacements are commonly used. The first, the generational replacement, replaces the entire population each generation [7]. The second, known as steady-state, only replaces a few individuals each generation $[29,31,32]$. In our experiments, initial population has been randomly generated and we use iteration limit exceeded as stopping criterion. Each population consists of 40 individuals, being replaced 4 of them each generation. Here we considered 250 generations. The selection phase allocates an intermediate population on the basis of the evaluation of the fitness function. We have chosen the stochastic universal selection scheme (SU) [11]. The crossover operator takes bits from each parent and combines them to create a child. Uniform crossover operator (U) is used here. It depends on the probability of exchange between two bits of the parents [28]. The mutation operator is better used after crossover [2]. It allows to reach individuals in the search space that could not be evaluated otherwise. When part of a chromosome has been randomly selected to be mutated, the corresponding genes belonging to that part are changed. This happens with probability $p$. This work deals with 
two mutation operators. The first is of the form $\nu \leftarrow \nu \pm p \times \nu$, where $\nu$ is the existing allele value, and $p$ is selected from a Gaussian distribution $(\mathrm{G})$. The second operator (R) simply replaces $\nu$ with a value selected uniformly randomly from the initialisation range of that gene.

The fitness function plays the role of the environment. It evaluates each string of a population. This is a measure, relative to the rest of the population, of how well that string satisfies a problem-specific metric. The values are mapped to a nonnegative and monotonically increasing fitness value. In the numerical experiments with this wind model [20], we look for optimal values of $\alpha, \varepsilon, \gamma$ and $\gamma^{\prime}$. Specifically, the so called stability parameter $\alpha$ determines the rate between horizontal and vertical wind adjustment. For $\alpha>>1$ flow adjustment in the vertical direction predominates, while for $\alpha<<1$ flow adjustment occurs primarily in the horizontal plane. Thus, the selection of $\alpha$ allows the air to go over a terrain barrier or around it. We search the optimum in $\left[10^{-2}, 10^{2}\right]$. The second parameter to be estimated is the weighting coefficient $\varepsilon(0 \leq \varepsilon \leq 1)$ involved in the horizontal interpolation of wind measurements. For $\varepsilon \rightarrow 1$, the importance of the horizontal distance from each point to the measurement stations is greater, while $\varepsilon \rightarrow 0$ signifies more importance of the height difference between each point and the measurement stations. The parameter $\gamma$ is related to the height of the planetary boundary layer. There exist different versions of where to search for this parameter. The interval $[0.15,0.4]$ considered in our simulations includes all the proposed search spaces. Finally, the parameter $\gamma^{\prime}$ appears in the computation of the mixing height for stable atmosphere. Several authors have proposed that the value of $\gamma^{\prime}$ should be searched in the surroundings of 0.4. More details and references about the discussion of these parameters can be found in [20].

We propose to minimise the following fitness function which is defined as the average relative error of the wind velocities given by the model with respect to the measures at the reference stations,

$$
F\left(\alpha, \varepsilon, \gamma, \gamma^{\prime}\right)=\frac{1}{N_{r}} \sum_{n=1}^{N_{r}} \frac{\left|\mathbf{v}_{n}-\mathbf{v}\left(x_{n}, y_{n}, z_{n}\right)\right|}{\left|\mathbf{v}_{n}\right|}
$$

where $\mathbf{v}\left(x_{n}, y_{n}, z_{n}\right)$ is the wind velocity obtained by the model at the location of station $n, \mathbf{v}_{n}$ is the measured wind and $N_{r}$ is the number of reference stations.

\section{Numerical Experiments}

We present several applications to show the improvements carried out in our wind model. All experiment were run on a XEON precision 530, except the parameter estimation problem which was solved using a cluster of PCs. 


\subsection{Surface Mesh Adaption to Orography and Roughness}

The studied three-dimensional domain is located in a region of Lugo, Spain, at $43 N$ of latitude and it is defined by four points of UTM coordinates $A(609980$, $4799020), B(626000,4799020), C(626000,4813040)$ and $D(609980,4813040)$, respectively. The height of the top is $4000 \mathrm{~m}$. A digital topographic map was provided by $D E S A$ on a mesh of element size $20 \times 20 \mathrm{~m}$. The $X$ axis corresponds to East direction and the $Y$ one to North. Thus, we are working with a region of $16020 \times 14020 \mathrm{~m}$. The minimum and maximum heights are $420 \mathrm{~m}$ and $1020 \mathrm{~m}$, respectively. Figure 1 represents the heights of the terrain. The measurement stations and the control points has been approximately plotted, such that from North to South we can find E243, E208, E212, E242, E206 and E283. Tables 3 and 4 contain their coordinates, respectively. Roughness is an essential factor on the atmospheric stratification, and therefore, on the characteristics of the resulting wind profile. Figure 2 shows the roughness length of the terrain which were supplied by DESA. We remark that some stations and control points are closed to contours of the roughness. In this case, the roughness length values are $0.03 \mathrm{~m}, 0.05 \mathrm{~m}, 0.08 \mathrm{~m}, 0.3 \mathrm{~m}$ and $0.8 \mathrm{~m}$.

\begin{tabular}{|c|c|c|c|}
\hline at & JTA & & \\
\hline$\overline{\mathrm{E} 2}$ & 615396 & 80 & \\
\hline & & & 945.0 \\
\hline פ2 & 617423 & 82 & 895.0 \\
\hline
\end{tabular}

Table 3. Coordinates in $m$ of the measurement stations.

\begin{tabular}{|c|c|c|c|}
\hline Jontr & UTI & 1 & Helg \\
\hline E24 & 618290 & 4806136 & $\overline{873.2}$ \\
\hline $\mathrm{E} 2$ & 616629 & 4808235 & 947.0 \\
\hline E283 & 617473 & 4804111 & 849.0 \\
\hline
\end{tabular}

Table 4. Coordinates in $m$ of the control points.

Starting from a regular mesh of the rectangular region with element size of $1 \times 1 \mathrm{~km}$ approximately, five global refinements are carried out using 4-T Rivara's algorithm [25]. With this number of refinement steps, we obtain a mesh with an element size about $31 \mathrm{~m}$. In order to improve the discretization near the stations and control points, five additional local refinements are applied inside six circles with centre at the stations and control points, respectively, and diameter $200 \mathrm{~m}$. This produces a local element size about $1 \mathrm{~m}$. Once we 


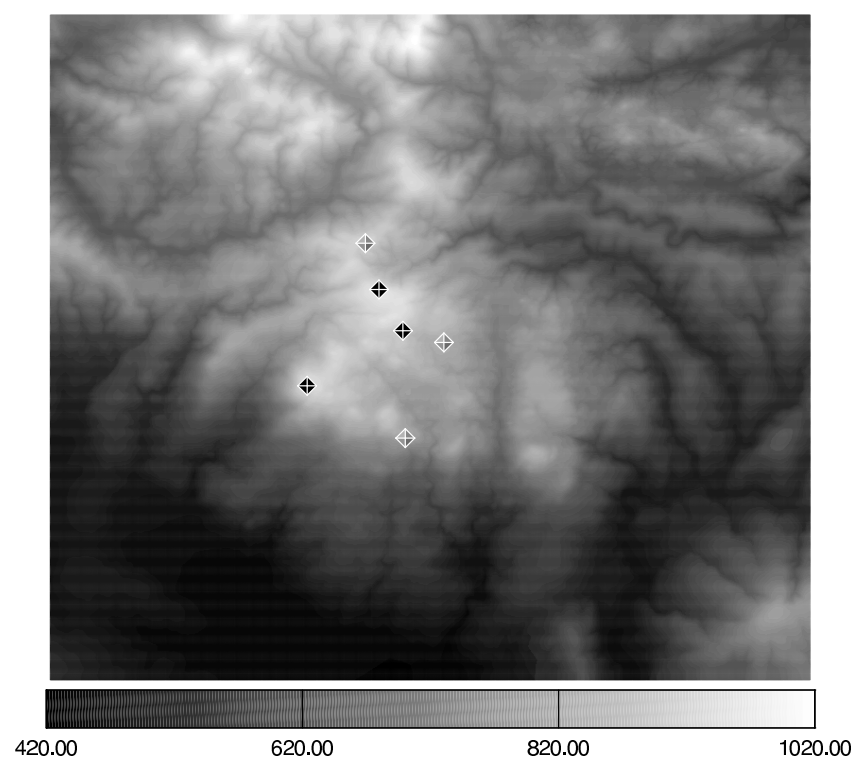

Fig. 1. Topographic map of the studied region in Lugo. From North to South, we can see the stations or control points E243, E208, E212, E242, E206 and E283.

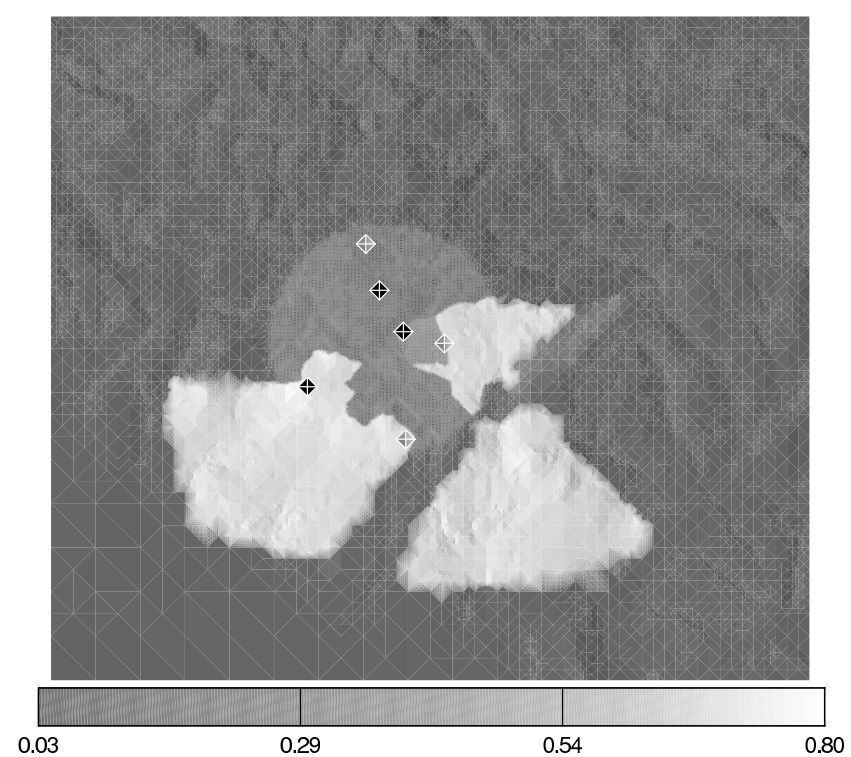

Fig. 2. Roughness length map of the studied region in Lugo with the station and control points. 
have interpolated the height and the roughness length in the nodes of these refined two-dimensional mesh, we use the derefinement algorithm $[5,24]$ described in section 2.1 with $\varepsilon_{h}=10$ and $\varepsilon_{r}=0.01$, keeping in any case the nodes located inside the circles. In figure 3 we can see the resulting triangulation of the terrain surface. The corresponding three-dimensional mesh, see figure 4, contains 102662 nodes and 515812 tetrahedra.

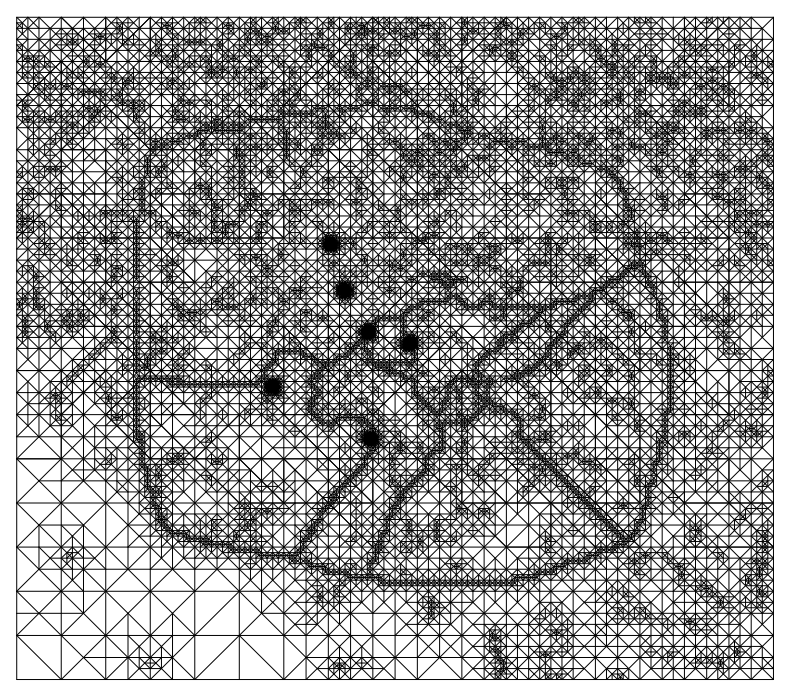

Fig. 3. Triangulation of the terrain simultaneously adapted to orography and roughness corresponding to the studied region in Lugo.

\subsection{Surface Mesh Adaption to Contours}

In many cases of environmental modelling, there are some contour lines which determinate certain characteristics of the studied region. For example, in wind simulation the well definition of shore lines or roughness contours may be very important for obtaining accurate results. Thus the mesh must be adapted following these contours such that they are represented by edges of the mesh. The procedure uses ideas from the smoothing of surface triangulation algorithm presented in [4]. As example, figure 5 shows an adaptive mesh, related to a region of the north west of Gran Canaria Island, to the shore line (plotted by points in black). 


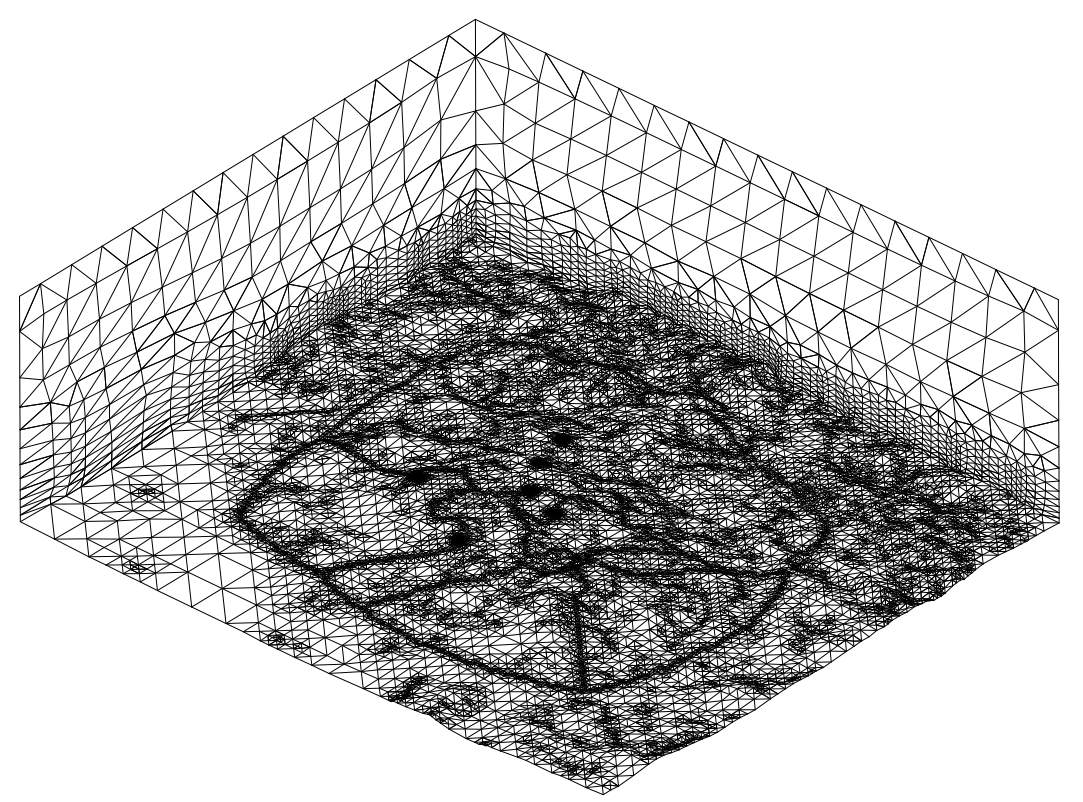

Fig. 4. Adaptive 3-D mesh corresponding to the studied region in Lugo.

\subsection{Wind Simulation in a Realistic Episode}

Using the mesh constructed for the Lugo application in the first numerical experiment, we have made a simulation for an episode along the March 21, 2003. The first step is to estimate the main parameters of the model and, then, apply the wind model using the estimated values. Next, the wind velocity is checked in the control points, where the wind data is supplied by DESA.

\section{Parameter Estimation Along a Day}

We have taken into account the table 2 for determining the stability class from the available turbulence intensity values. For the studied day, we have obtained neutral conditions. So we must estimate the stability parameter $\alpha$, the weighting parameter $\varepsilon$ related to the horizontal interpolation of wind velocities and the parameter $\gamma$ involved in the computation of the planetary boundary layer; see, e.g., [20]. The estimation has been carried out each hour (24 computations). We have applied genetic algorithms to solve these parameter estimation problems, where the fitness functions are defined in terms of the relative velocity errors obtained by the model at the measurements stations. It is evident that, in order to avoid spurious solutions, more than 20 repetitions for parameter setting of each hour should be done. This fact would obviously imply an important increasing on the computational cost of the parameter 


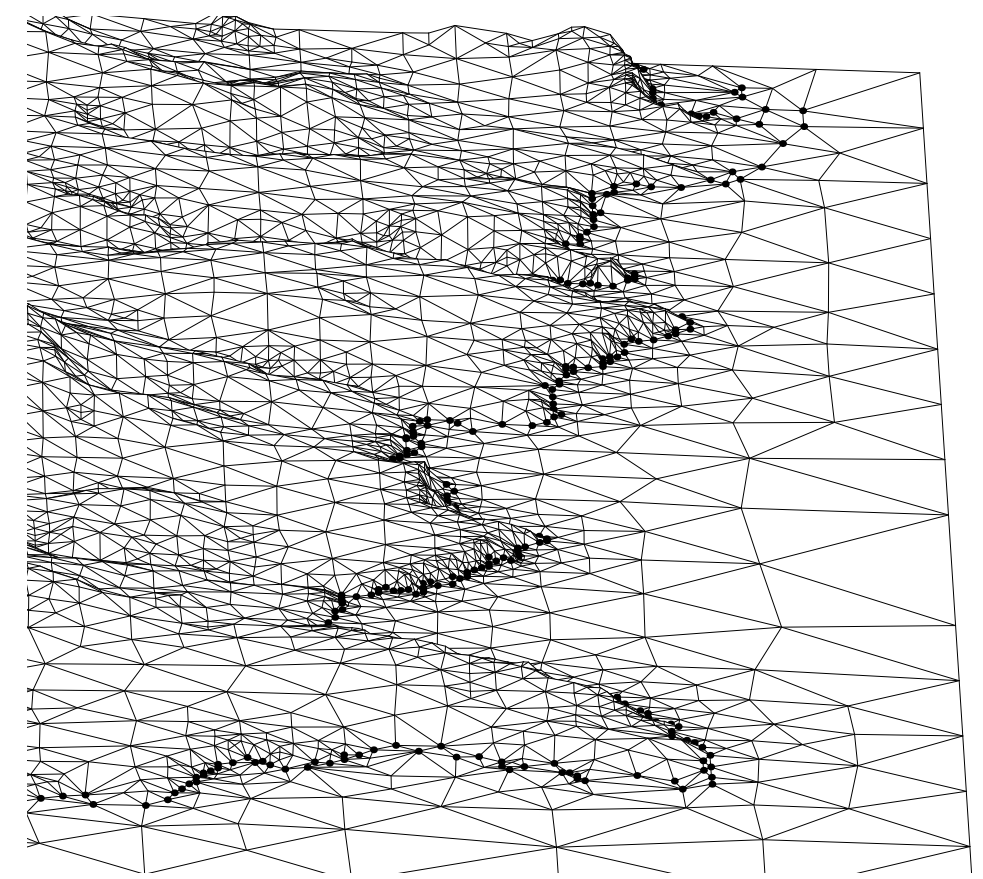

Fig. 5. Region defined in the north west of Gran Canaria Island. Contour plots and adapted mesh.

estimation process, even more if we take into account that each evaluation of the fitness function supposes the resolution of a finite element problem (in this case, about one hundred thousand unknowns). Nevertheless, from our previous experience in this kind of wind simulation problems, we have observed that just one computation is enough for reaching a good solution.

In figure 6 we can see the evolution of the values of the three parameters along the episode. The values of $\varepsilon$ are practically constant and approximately equal to 1 . This means that only the horizontal distance has effect on the horizontal interpolation. This result is agreed with the orographic characteristics of the studied domain. Likewise the values obtained for $\gamma$ are closed to 0.15, that is, the lower limit for this parameter which is related to low planetary boundary layers. However, the stability parameter $\alpha$ varies in the interval 8 20. This range of values makes the wind predominantly flow more over the obstacles than around them.

\section{Comparison of the model results with empirical data}

Once the main parameters are estimated, we start the wind modelling along the selected episode using the obtained values. For March 21, 2003, only measures from E242 and E283 were available. Figures 7 and 8 show the wind 


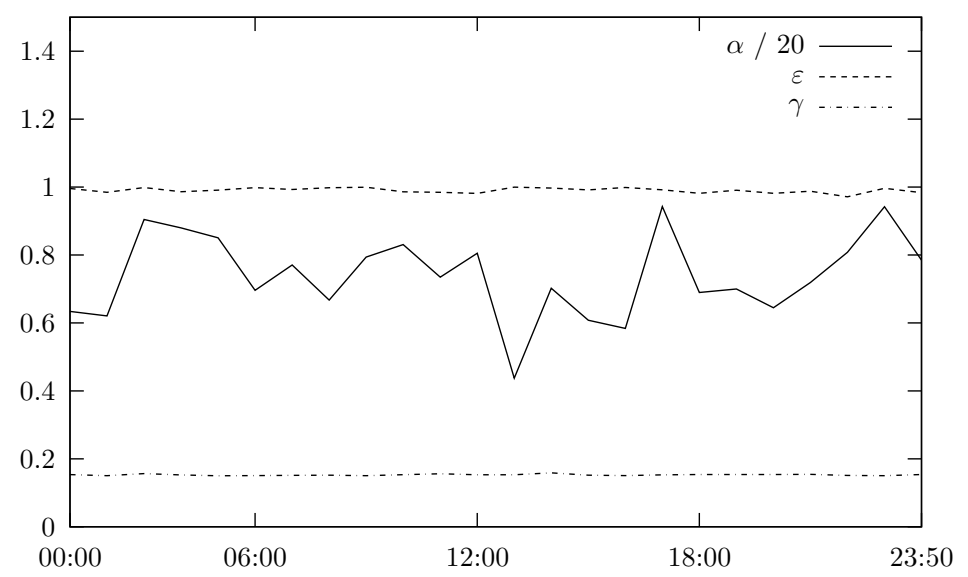

Fig. 6. Results of the estimation of $\alpha, \varepsilon$ and $\gamma$ along the studied episode (March 21, 2003)

speeds obtained with the model and the reference values measured at the control points E242 and E283, respectively. More details of the errors of computed winds with respect to the measured wind may be seen in table 5 . We remark that the average errors at the measurement stations are small as expected. The average error is $27.24 \%$ at control point E242 and $4.94 \%$ at E283.

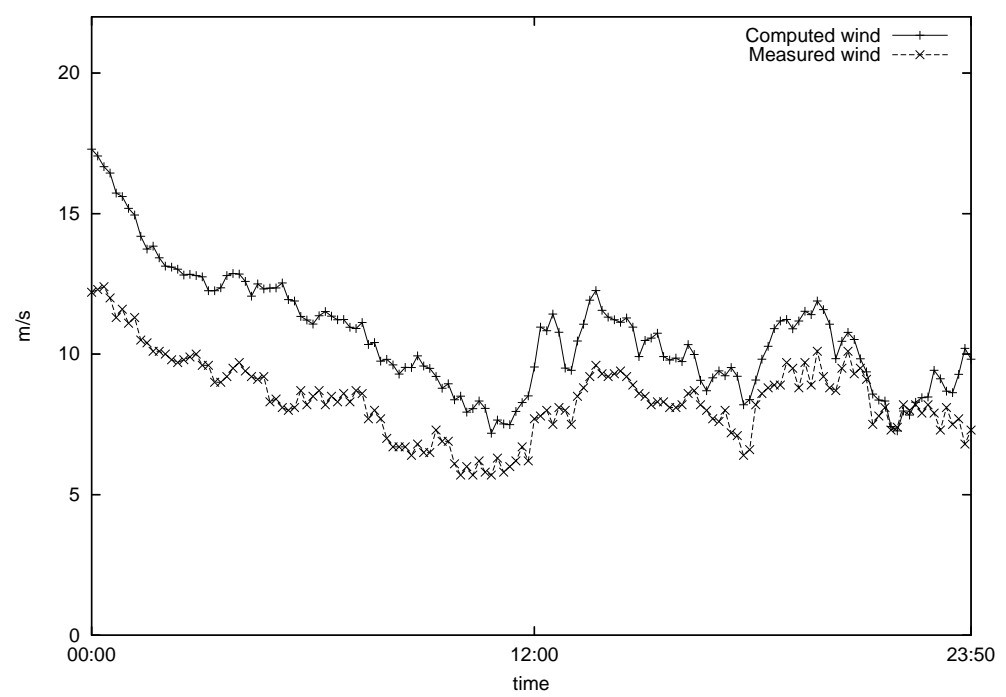

Fig. 7. Comparison of the wind velocities measured at the control station E242 (March 21, 2003). 


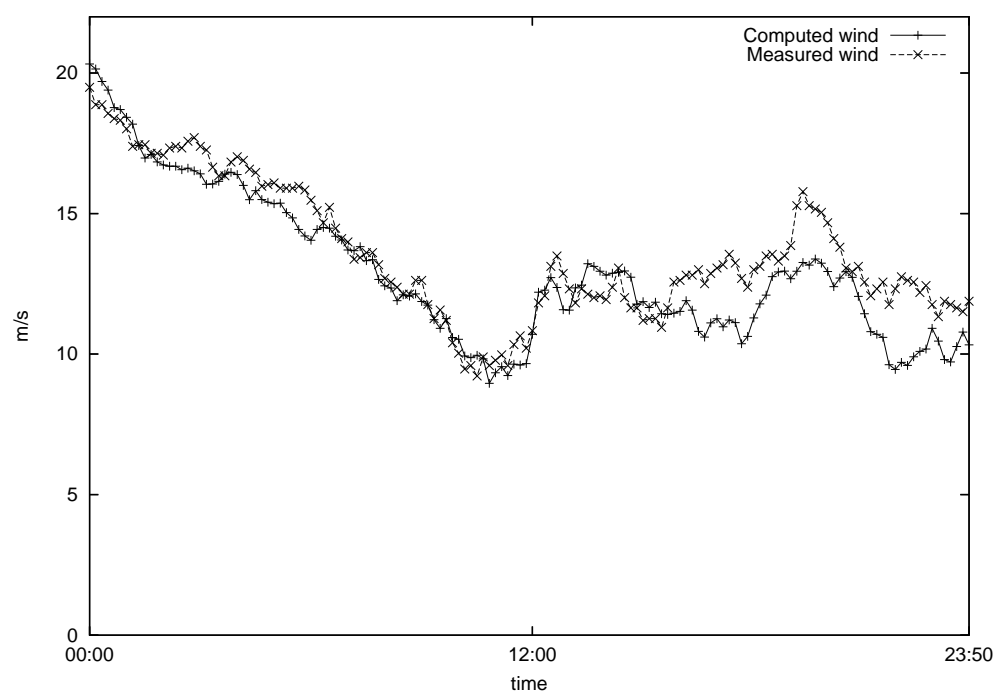

Fig. 8. Comparison of the wind velocities measured at the control station E283 (March 21, 2003).

\begin{tabular}{|c|c|c|c|c|c|}
\hline $\begin{array}{l}\text { Stations and } \\
\text { control points }\end{array}$ & $\begin{array}{c}\text { Average } \\
\text { measured } \\
\text { wind }\end{array}$ & $\begin{array}{c}\text { Average } \\
\text { computed } \\
\text { wind }\end{array}$ & $\begin{array}{c}\% \\
\text { d average } \\
\text { error }\end{array}$ & $\begin{array}{l}\text { Maximum } \\
\text { absolute } \\
\text { error }\end{array}$ & $\begin{array}{c}\text { Minimum } \\
\text { absolute } \\
\text { error }\end{array}$ \\
\hline$\overline{\mathrm{E}} \mathrm{E} 206(49 \mathrm{~m})$ & 15.37 & 15.50 & $0.81 \%$ & 0.46 & 0.01 \\
\hline $\mathrm{E} 208(15 \mathrm{~m})$ & 8.5 & 8.98 & $4.74 \%$ & 1.25 & 0.00 \\
\hline $\mathrm{E} 208(30 \mathrm{~m})$ & 9.2 & 9.92 & $7.21 \%$ & 1.36 & 0.05 \\
\hline $\mathrm{E} 212(15 \mathrm{~m})$ & 8.46 & 8.44 & $0.20 \%$ & 0.63 & 0.00 \\
\hline $\mathrm{E} 212(30 \mathrm{~m})$ & 9.02 & 9.85 & $9.25 \%$ & 1.60 & 0.31 \\
\hline $\mathrm{E} 242(40 \mathrm{~m})$ & 8.40 & 10.69 & $27.24 \%$ & 5.09 & 0.09 \\
\hline $\operatorname{E} 283(49 m)$ & 13.62 & 12.95 & $4.94 \%$ & 3.04 & 0.02 \\
\hline
\end{tabular}

Table 5. Error of the computed wind at stations and control points.

\section{Conclusions}

We have proposed a technique for constructing tetrahedral meshes which are simultaneously adapted to the terrain orography and the roughness length. The use of our refinement/derefinement process in the 2-D mesh corresponding to the terrain surface allows us to obtain meshes that are accurately adapted 
to different functions as well as are locally refined around several points. These characteristics of the generated meshes are very important in the wind simulation since, on the one hand, the quality of the representation of both orography and roughness is critical for obtaining accurate results with the model, and on the other hand, the local refinement at the stations and control points is essential for inserting the wind data of the stations or recovering such data at any required point.

Some improvements have been carried out in the construction of the initial wind based on the horizontal interpolation of wind measures and vertical extrapolation in stratified atmosphere. The optimization of the friction velocity for several measures in the same tower allows to minimize the differences between the constructed vertical profile of wind and the measures. However, though such differences are small, further research is needed in order to construct new wind profiles that exactly satisfy all the available measures of wind velocities. In addition, the inclusion of observations of turbulence intensities has made the model to be able of automatically updating the suitable wind profile as function of the corresponding stability class.

The periodic updating of the main parameters of the model has proved to be fundamental for reducing the errors of the computed wind. However, further considerations should be taken into account in future works for a better performance of the model. For example, a finer map of roughness, a more sophisticated horizontal interpolation of wind velocities and a greater number of measurement stations well distributed over the studied region, will help to reduce errors at points like E242 where the roughness may not be well approximated. Finally, in order to obtain an accurate wind field in zones with very steep slopes, the mesh should be adapted to the contour lines, since a change in the direction of edges in the mesh may strongly affect the computed wind.

\section{Acknowledgement}

The work has been partially supported by the Spanish Government (Ministerio de Educación y Ciencia) and FEDER, grant number CGL2004-06171C03-02/CLI. The authors are also grateful to Ignacio Láinez and Antonio Ruiz for their technical support provided under the scope of the collaboration agreement signed by the University of Las Palmas de Gran Canaria and Desarrollos Eólicos (DESA) in January 2005. The wind data correspond to a DESA's wind farm located in Lugo.

\section{References}

1. Bäck T, Fogel DB, Michalewicz Z (1997) Handbook of evolutionary computation. Oxford Univ. Press, New York-Oxford

2. Davis L (1991) Handbook of genetic algorithms. Van Nostrand Reinhold 
3. Escobar JM, Rodríguez E, Montenegro R, Montero G, González-Yuste JM (2003) Simultaneous untangling and smoothing of tetrahedral meshes. Comp Meth Appl Mech Eng 192:2775-2787

4. Escobar JM, Montero G, Montenegro R, Rodríguez E (2006) An algebraic method for smoothing surface triangulations on a local parametric space. Int J Num Meth Eng 66:740-760

5. Ferragut L, Montenegro R, Plaza A (1994) Efficient refinement/derefinement algorithm of nested meshes to solve evolution problems. Comm Num Meth Eng 10:403-412

6. González-Yuste JM, Montenegro R, Escobar JM, Montero G, Rodríguez E (2004) Local refinement of 3-D triangulations using object-oriented methods. Adv Eng Soft 35:693-702

7. Holland J (1992) Adaption in natural and artificial systems. MIT Press

8. Kitada T, Kaki A, Ueda H, Peters LK (1983) Estimation of vertical air motion from limited horizontal wind data - A numerical experiment. Atmos Environ $17: 2181-2192$

9. Lalas DP, Tombrou M, Petrakis M (1988) Comparison of the performance of some numerical wind energy siting codes in rough terrain. In: European Community Wind Energy Conference, Herning, Denmark

10. Lalas DP, Ratto CF (1996) Modelling of atmospheric flow fields. World Scientific Publishing, Singapore

11. Levine D (1994) A Parallel Genetic Algorithm for the Set Partitioning Problem. $\mathrm{PhD}$ Thesis, Illinois Institute of Technology / Argonne National Laboratory

12. Michalewicz Z (1994) Genetic algorithms + data structures = evolution problems. Springer Verlag, Berlin-Heidelberg-New York

13. Mikkelsen T (2003) Modelling of pollutant transport in the atmosphere. MANHAZ position paper, Ris $\emptyset$ National Laboratory, Denmark

14. Montenegro R, Montero G, Escobar JM, Rodríguez E, González-Yuste JM (2002) Tetrahedral mesh generation for environmental problems over complex terrain. Lect N Comp Sci 2329:335-344

15. Montenegro R, Montero G, Escobar JM, Rodríguez E (2002) Efficient strategies for adaptive 3-D mesh generation over complex orography. Neural, Parallel \& Scientific Computation 10:57-76

16. Montero G, Montenegro R, Escobar JM (1998) A 3-D diagnostic model for wind field adjustment. J Wind Engrg Ind Aer 74-76:249-261

17. Montero G, Sanin N (2001) Modelling of wind field adjustment using finite differences in a terrain conformal coordinate system. J Wind Engrg Ind Aer 89:471-488

18. Montero G, Montenegro R, Escobar JM, Rodríguez (2003) Generación automática de mallas de tetraedros adaptadas a orografías irregulares. Rev Int Mét Num Cálc Dis Ing 19(2):127-144

19. Montero G, Montenegro R, Escobar JM, Rodríguez E, González-Yuste JM (2004) Velocity field modelling for pollutant plume using 3-D adaptive finite element method. Lect N Comp Sci 3037:642-645

20. Montero G, Rodríguez E., Montenegro R, Escobar JM, González- Yuste JM (2005) Genetic algorithms for an improved parameter estimation with local refinenent of tetrahedral meshes in a wind model. Adv Engrg Soft 36:3-10

21. Moussiopoulos N, Flassak Th, Knittel G (1998) A refined diagnostic wind model. Environ Soft 3:85-94 
22. Pennel WT (1983) An Evaluation of the Role of Numerical Wind Field Models in Wind Turbine Siting. Batelle Memorial Institute, Pacific Northwest Laboratory, Richland, Washington

23. Pielke R (1984) Mesoscale meteorological modeling. Academic Press, Inc., Orlando, Florida

24. Plaza A, Montenegro R, Ferragut L (1996) An improved derefinement algorithm of nested meshes. Adv Eng Soft 27:51-57

25. Rivara MC (1987) A grid generator based on 4-triangles conforming. Meshrefinement algorithms. Int J Num Meth Eng 24:1343-1354

26. Rodríguez E, Montero G, Montenegro R, Escobar JM, González- Yuste JM (2002) Parameter estimation in a three-dimensional wind field model using genetic algorithms. Lect Notes in Comp Sci 2329:950-959

27. Seinfeld JH, Pandis SN (1998) Atmospheric chemistry and physics. From air pollution to climate change. John Wiley \& Sons, Inc., New York

28. Spears W, DeJong K (1991) On the virtues of parametrized uniform crossover. In: Proceedings of the Fourth International Conference on Genetic Algorithms

29. Syswerda G (1989) Uniform crossover in genetic algorithms. In: Proceedings of the Third International Conference on Genetic Algorithms

30. Vose M (1999) The simple genetic algorithm. MIT Press, Cambridge, Massachusetts

31. Whitley D (1988) GENITOR: A different genetic algorithm. In: Rocky Mountain Conference on Artificial Intelligence

32. Whitley D (1989) The GENITOR algorithm and selection pressure: Why rankbased allocation of reproductive trials is best. In: Proceedings of the Third International Conference on Genetic Algorithms

33. Winter G, Montero G, Ferragut L, Montenegro R (1995) Adaptive strategies using standard and mixed finite elements for wind field adjustment. Solar Energy $54: 49-56$ 